\title{
Offsetting virulence and antibiotic resistance costs by MRSA
}

\author{
James Collins ${ }^{1}$, Justine Rudkin ${ }^{2}$, Mario Recker ${ }^{1}$, Clarissa Pozzi ${ }^{3}$, James P O’Gara ${ }^{3}$ and \\ Ruth C Massey ${ }^{2}$ \\ ${ }^{1}$ Department of Zoology, University of Oxford, Oxford, UK; ${ }^{2}$ Department of Biology and Biochemistry, \\ University of Bath, Somerset, UK and ${ }^{3}$ School of Biomolecular and Biomedical Sciences, University College \\ Dublin, Dublin, Ireland
}

\begin{abstract}
The prevalence of diverse MRSA (methicillin-resistant Staphylococcus aureus) types in both hospital and community settings is a major health problem worldwide. Here we compare hospitalacquired MRSAs with large type II SCCmec elements with those prevalent in both hospital and community settings with smaller type IV SCCmec elements. We find that the type II but not the type IV SCCmec element causes the bacteria to reduce their levels of costly toxin expression. We compare the relative growth rates of these MRSA types and show that the type II SCCmec carrying MRSAs are more affected than those carrying type IV elements and from this we hypothesize that offsetting the costs associated with antibiotic resistance and toxin expression is why the type II are confined to hospital environments where antibiotic use, the prevalence of immunocompromised individuals and vector-mediated transmission is high. In contrast, those MRSAs that are also successful in the community can maintain their high levels of toxin expression due to a lower fitness burden associated with the smaller SCCmec element.
\end{abstract}

The ISME Journal (2010) 4, 577-584; doi:10.1038/ismej.2009.151; published online 14 January 2010

Subject Category: microbial ecology and functional diversity of natural habitats

Keywords: Staphylococcus aureus; MRSA; fitness costs; virulence costs

\section{Introduction}

The prevalence and success of MRSA (methicillinresistant Staphylococcus aureus) in health-care and community settings have resulted in much research into its pathogenicity in the hopes of identifying novel means of control. In the UK, $2 \%$ of hospital admissions become infected with $S$. aureus and of those approximately $60 \%$ will be methicillin resistant (Emmerson et al., 1996; Jones, 2003). Of increasing concern is the emergence of MRSA outside health-care settings among healthy individuals. In 2005 a study of invasive MRSA infections in nine US centers found that $13.7 \%$ were community acquired (Klevens et al., 2007), showing the emergence of this organism as a threat both in and outside hospitals.

The types of infections caused by $S$. aureus vary widely in both severity and site of infection. They can be relatively minor skin and soft tissue abscesses, life-threatening cases of septicemia or endocarditis, or chronic cases of osteomyelitis (Lowy, 1998; Gordon and Lowy, 2008). The genes

Correspondence: RC Massey, Department of Biology and Biochemistry, University of Bath, Somerset, UK.

E-mail: r.c.massey@bath.ac.uk

Received 20 October 2009; revised 30 November 2009; accepted 3 December 2009; published online 14 January 2010 expressed by $S$. aureus facilitating such varied infections are not fully understood, but can be grouped into three based on their function: the adhesins, the toxins and the immunomodulators. The adhesins are surface-attached proteins that allow the bacteria to attach to a wide variety of human tissues. The toxins are secreted proteins that cause tissue damage, and generate pus in abscesses, which is believed to facilitate transmission between hosts. The immunomodulators are proteins that interfere with host immunity preventing defense against, and clearance of, infections. The expression of these three groups of proteins is critical in determining the success of an infection.

Methicillin resistance is conferred by the acquisition of a piece of DNA referred to as the SCCmec element (Deurenberg et al., 2007). The size and content of this element vary and is currently classified into five major groups (SCCmec types $\mathrm{I}-\mathrm{V}$ ) based on their content (Deurenberg et al., 2007). There are two features common to all SCCmec elements: the mecA gene, which encodes the alternative penicillin-binding protein that confers methicillin resistance, and the $c c r$ recombination sites and associated recombinases that cause this mobile piece of DNA to excise and transmit horizontally (Hiramatsu et al., 2001). SCCmec elements I-III are larger than the others and are predominantly found causing hospital- and health-care-associated 
infections. SCCmec types IV and V are not restricted to these environments and can cause infections in otherwise healthy individuals with no health-care contact (Ito et al, 2004; Ma et al., 2002). Although many individual virulence factors have been found to be associated with community-acquired MRSA (for example cytolytic proteins (Wang et al., 2007), $\alpha$-toxin (Li et al., 2009) and the PVL leukotoxin (Labandeira-Rey et al., 2007), it is still not clear why these strains are successful both in and outside health-care settings.

Antibiotic resistance is often associated with a fitness cost (Andersson and Levin, 1999), but compensatory mutations can arise elsewhere in the bacterial genome that alleviates some or all of these costs (Maisnier-Patin and Andersson, 2004). Previous studies have shown that the relative fitness of hospital-acquired MRSAs carrying the type I SCCmec element is reduced (Ender et al., 2004), whereas strains containing the smaller type IV element were not affected (Lee et al., 2007). It has also been shown in vancomycin-resistant MRSA strains containing the type II SCCmec element that deletion of mecA compensated for some of the fitness costs associated with becoming vancomycin resistant, suggesting that mecA is also costly (Noto et al., 2008). A detailed study of the costs associated with all five classes of SCCmec elements and whether these costs have been compensated in clinical strains has not been performed to date.

Work by us and others have reported diverse virulence phenotypes among MRSA and MSSA (methicillin-sensitive Staphylococcus aureus) strains (Collins et al., 2008; Fowler et al., 2004; Vaudaux et al., 1998; O'Neill et al., 2008). Here we examine the ability of both clinical and isogenic MSSAs and MRSAs with the large type II (hospital-associated) and smaller type IV (hospital- and communityassociated) SCCmec elements to lyse cells. We find that the type II SCCmec carrying MRSA strains have reduced toxicity. A measure of the relative fitness of these strains shows that the type II SCCmec carrying MRSA strains also have a significant fitness burden associated with them. We hypothesize that offsetting costly toxin expression with antibiotic resistance may explain the prevalence of these different MRSA type in diverse environment and test this using a mathematical model.

\section{Materials and methods}

T-cell toxicity assay

T2 cells (immortalized T-cell line, Salter and Cresswell, 1986) were propagated in T-cell media (50 ml aliquots of RPMI, supplemented with $10 \%$ heat-inactivated fetal bovine serum, $1 \mathrm{~mm}$ L-glutamine, $200 \mathrm{U} \mathrm{ml}^{-1}$ penicillin and $0.1 \mathrm{mg} \mathrm{ml}^{-1}$ streptomycin, at $37^{\circ} \mathrm{C}$ in a humidified incubator with $5 \%$ $\mathrm{CO}_{2}$ in air) for $48 \mathrm{~h}$ and then harvested by centrifugation for $5 \mathrm{~min}$ at $700 \mathrm{~g}$, and gently washed and resuspended in $1 \mathrm{ml}$ warm tissue culture-grade phosphate-buffered saline. Washing was repeated to ensure all antibiotics were removed. T cells were diluted in phosphate-buffered saline to a final cell concentration of $3 \times 10^{6} \mathrm{ml}^{-1}$. Cells $(15 \mu \mathrm{l})$ were transferred to a sterile microfuge tube for each assay undertaken. The bacteria (strains used are described in the Supplementary Table) were grown for $17 \mathrm{~h}$ in $5 \mathrm{ml}$ BHI broth, $750 \mu \mathrm{l}$ of culture was removed, centrifuged for $2 \mathrm{~min}$ and the supernatant was harvested. Bacterial supernatant $(15 \mu \mathrm{l})$ and washed $\mathrm{T}$ cells $(15 \mu \mathrm{l})$ were incubated for $10 \mathrm{~min}$. Cell death was measured using $15 \mu \mathrm{l}$ Trypan blue (Sigma) and a Fast-Read counting chamber (Immune Systems, Paignton, UK). Each clinical strain indicated in the Supplementary Table was assayed once as each was considered a biological replicate indicative of its group; for the isogenic strains 10 independently grown cultures were assayed.

\section{Relative fitness assay}

Bacteria (strains used are described in the Supplementary Table) were grown overnight in BHI broth to an $\mathrm{OD}_{600}$ of 2.0 to ensure that all cells are in a similar physiological state at the start of the experiment. Competitions were established in either $0.25 \mathrm{X}$ BHI broth or spent broth (BHI broth in which $S$. aureus strains RN4390B was grown overnight, the bacteria removed by centrifugation and the medium was filter sterilized). The competition medium was inoculated with $10^{3}$ c.f.u. per ml of the marker strain (MSSA466 which is tetracycline resistant) and the test strain. Initial cell numbers were confirmed by plating. The bacteria were competed at $37^{\circ} \mathrm{C}$ in a shaking incubator (180 r.p.m.) for $24 \mathrm{~h}$. Final cell numbers were enumerated by serial dilutions on TSA plates (total cell count), and TSA plates containing $2 \mu \mathrm{g} \mathrm{ml}^{-1}$ tetracycline (marker strain count). The fitness of a strain was defined as a measure of the reproductive success of the population, which can be expressed as the natural logarithm of the ratio of the final and initial cell densities of the culture (Lenski et al., 1991). Each clinical strain was assayed once as each was considered a biological replicate indicative of its group; for the isogenic strains 10 independently grown cultures were assayed.

$$
\text { relative (Darwinian) fitness }=\frac{\ln (A(1) / A(0))}{\ln (M(1) / M(0))}
$$

where: $A(0)$, estimated density of test strain at time $0 ; M(0)$, estimated density of marker strain at time 0 ; $A(1)$, estimated density of test strain at time 1 day; $M(1)$, estimated density of marker strain at time 1 day; ln, natural logarithm (logarithm to the base $e$ ).

\section{Construction of BH1CC $\triangle S C C$ mec mutant}

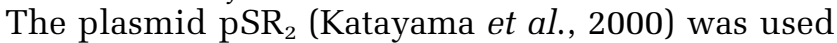
to excise the SCCmec cassette from the methicillinresistant $S$. aureus chromosome. The vector $\mathrm{pSR}_{2}$ 
contain the genes $c c r A$ and $c c r B$ encoding polypeptides having a partial homology to recombinases of the invertase/resolvase family. The open reading frames were found to catalyze precise excision of the SCCmec element. The vector which has a temperature-sensitive origin of replication (one copy at $37^{\circ} \mathrm{C}$ ) was introduced into BH1CC by electroporation. The strain was subcultured in drug-free broth for 7 nights at $37^{\circ} \mathrm{C}$ and the proportion of colonies that had lost the SCCmec was evaluated by plating onto agar plates with and without oxacillin and by PCR analysis.

\section{Statistics}

T-cell toxicity data contained a number of zero values and as such it was not possible to normalize. The nonparametric Mann-Whitney test was used to determine a statistical difference between groups.

The relative fitness data were determined to be normal using the Anderson-Darling normality test. Statistical differences between groups were determined using a two-tailed $t$-test. Tests between groups were carried out on different days (that is MRSA/ MSSA CC30 strains were tested separately from MRSA/MSSA CC22 and CC8 strains); it is therefore not reliable to test between these groups as small differences in the initial marker strain could cause a statistical effect. Where tests have been carried out, relative fitness tests were carried out at the same time, using the same media, and the same marker strain.

\section{Mathematical modeling}

The model describes the dynamics of three $S$. aureus strains competing within the population: MSSA (methicillin susceptible), IV-MRSA (type IV SCCmec carrying MRSA) and II-MRSA (type II SCCmec carrying MRSA). It is assumed that susceptible hosts, $S$, can only be infected by one strain at the time on which they move into the infected classes, $I_{\mathrm{MSSA}}, I_{\mathrm{IV}-\mathrm{MRSA}}$ or $I_{\mathrm{II}-\mathrm{MRSA}}$; after successful treatment infected individuals return to the susceptible class, that is infection does not offer immunity to renewed infection. Infection itself has two components/ routes: one is by direct host-host contact and to a degree dependent on the production of toxins, the other is due to secondary contact, for example through health-care workers within a community or hospital environment.

The model can then be given as the following set of differential equations describing the rate of change in the proportion of the population currently susceptible or infected:

$$
\begin{aligned}
\frac{\mathrm{d} S}{\mathrm{~d} t} & =\mu-S \sum_{i} \lambda_{i}-\mu S+\sum_{i} \tau_{i} \\
\frac{\mathrm{d} I_{i}}{\mathrm{~d} t} & =\lambda_{i} S-\left(\mu+\delta_{i}+\tau_{i}\right) I_{i}, \\
i & =\text { MSSA, II-MRSA, IV-MRSA }
\end{aligned}
$$

where $\mu(=0.013)$ is the average host birth and death rate (we assume constant population size); $\tau_{i}$ and $\delta i$,
Table 1

\begin{tabular}{lcccccc}
\hline Strain (i) & $\beta_{t}^{i}$ & $\beta_{h}{ }^{\mathrm{a}}$ & $\mathrm{C}_{t}^{i}$ & $\mathrm{C}_{r}^{i}$ & $\delta_{i}$ & \multicolumn{1}{c}{$\tau_{i}^{\mathrm{a}}$} \\
\hline MSSA & 0.3 & $0.1 / 1$ & 0.2 & 0 & 0.01 & $0.2 / 0.5$ \\
IV-MRSA & 0.4 & $0.1 / 1$ & 0.2 & 0.1 & 0.02 & $0.1 / 0.25$ \\
II-MRSA & 0.2 & $0.1 / 1$ & 0 & 0.1 & 0.02 & $0.1 / 0.25$ \\
\hline
\end{tabular}

${ }^{\mathrm{a}}$ (community/hospital).

the rate of treatment- and infection-associated mortality of strain $i$ respectively.

The force of infection of strain $i$ is given as:

$$
\lambda_{\mathrm{i}}=I_{i}\left(\beta_{\mathrm{t}}^{i}+\beta_{\mathrm{h}}\right)\left(1-c_{\mathrm{r}}^{i}\right)\left(1-c_{t}^{i}\right)
$$

where $\beta_{\mathrm{i}}^{i}$ and $\beta_{\mathrm{h}}$ are the transmission parameters through direct and secondary (that is vectormediated) contact respectively, and $C_{\mathrm{i}}^{i}$ and $C_{\mathrm{r}}^{i}$ the respective fitness costs associated with toxin production and antibiotic resistance. Note, secondary transmission is strain independent and will be used to describe the two different transmission environments: community and hospital.

The main assumption/parameter values are given in the following table 1 :

This means, we assume that the propensity of MSSA to cause transmissible illness/disease is greater than for II-MRSA as these have reduced toxin production, which is also reflected in a reduced fitness cost, that is $\beta_{t}^{\mathrm{MSSA}}>\beta_{t}^{\text {I-MRSA }}$ and $c_{t}^{\mathrm{MSSA}}>C_{\mathrm{t}}^{\mathrm{II}-\mathrm{MRSA}}$. IV-MRSA on the other hand is even more transmissible than MSSA but at no extra fitness cost, that is $\beta_{t}^{\mathrm{IV}-\mathrm{MRSA}}>\beta_{t}^{\mathrm{MSSA}}$ and $c_{t}^{\mathrm{MSSA}}=c_{t}^{\mathrm{NV}-}$ MRSA. We furthermore assume that infections by methicillin-resistant strains have a reduced treatment success rate and therefore a (slightly) higher chance of being lethal, although the latter assumption is not a necessary for the results presented here. We distinguish between two different environments: (1) a community setting where the majority of transmission is through direct, rather than vectormediated, contact; and (2) a hospital setting with a greatly enhanced chance of vector-mediated transmission but also a higher rate of treatment.

The basic reproductive ratios of the three strains are given as

$$
\begin{aligned}
R_{0}^{i} & =\frac{\left(\beta_{t}^{i}+\beta_{t}^{a}\right)\left(1-c_{t}^{i}\right)\left(1-c_{r}^{i}\right)}{\mu+\delta_{i}+\tau_{i}^{a}}, \\
i & =\text { MSSA, II - MRSA, IV - MRSA }
\end{aligned}
$$

We can then find the condition under which either type II or type IV-MRSA dominate the population simply as $R_{0}^{\text {III-MRSA }}>R_{0}^{\text {IV-MRSA }}>R_{0}^{\mathrm{MSSA}}$ or $R_{0}^{\mathrm{IV}-\mathrm{MRSA}}>R_{0}^{\mathrm{II}-}$ $\mathrm{MRSA}^{\prime}>R_{0}^{\mathrm{MSSA}}$ respectively. Under the assumption that the cost of resistance, rate of treatment and induced mortality are equal for type II and type IV MRSAs, we can also find an explicit condition for type II outcompeting type IV MRSA as

$$
\begin{aligned}
& R_{0}^{\mathrm{II}-\mathrm{MRSA}}>R_{0}^{\mathrm{IV}-\mathrm{MRSA}} \Leftrightarrow\left(\beta_{t}^{\mathrm{II}-\mathrm{MRSA}}+\beta_{t}^{a}\right)\left(1-c_{t}^{\mathrm{II}-\mathrm{MRSA}}\right) \\
& >\left(\beta_{t}^{I V-M R S A}+\beta_{t}^{a}\right)\left(1-c_{t}^{\mathrm{IV}-\mathrm{MRSA}}\right),
\end{aligned}
$$


and vice versa. This can simply be interpreted as follows: if the cost of toxin production cannot be offset by a gain in transmissibility (in different environments), then a particular strain will be outcompeted and replaced by another strain with either a lower fitness cost or higher transmissibility.

\section{Results}

Carriage of the type II but not the type IV SCCmec element affects MRSA toxicity

Hospital-acquired MRSA strains, such as those containing the type II SCCmec element, rarely cause disease outside health-care setting among healthy people (Hiramatsu et al., 2001). This has been partially attributed to diminished growth rates associated with antibiotic resistance, but our findings suggest that lower levels of toxicity may also contribute to reduced virulence in healthy individuals (Collins et al., 2008). MRSA strains containing the smaller type IV SCCmec element are successful both in and outside health-care settings, suggesting that these MRSA strains may be more toxic than strains carrying the type II element. To test this, we compared the toxicity of both clinical and isogenic type II and type IV SCCmec carrying MRSA strains. Figure 1 shows that the type II SCCmec element reduces the toxicity of strains and shows that carriage of the type IV element has no such effect.
Toxin expression and the type II SCCmec element affect the relative fitness of $\mathrm{S}$. aureus

We hypothesize that the smaller type IV SCCmec element may have less of a fitness burden associated with it. To test this, we performed competitions in low-nutrient broth $(0.25 \mathrm{X}$ BHI) against a marked MSSA strain (this medium was selected to provide the optimum nutrient level to get direct competition for nutrients between the marked and tester strains as well as sufficient growth of the bacteria for fitness differences to be detectable). The relative fitness of clonally matched clinical MSSA and MRSA strains (containing both type II and IV SCCmec elements) and isogenic type II and IV MRSA/MSSA pairs was measured (Figure 2). For both the clinical and isogenic strains the type II SCCmec element reduced relative fitness of the strain carrying it $(P<0.001$, $T=6.08, \quad$ d.f. $=57$ and $P=0.0044, \quad T=-3.28$, d.f. $=17$ respectively). The type IV SCCmec carrying clinical strains were slightly less fit than MSSA strains from the same clonal complexes (CC8 and $22)$ that just reached statistical significance $(P=0.049, \quad T=2.32$, d.f. $=8)$ but there was no difference in fitness between the isogenic pair of strains $(P=0.64, \quad T=-0.47, \quad$ d.f. $=26)$ showing that this element does not incur a fitness cost in this experimental system. In all cases, a two-sample $t$-test was used to determine a statistical difference as the data were shown to be normal.

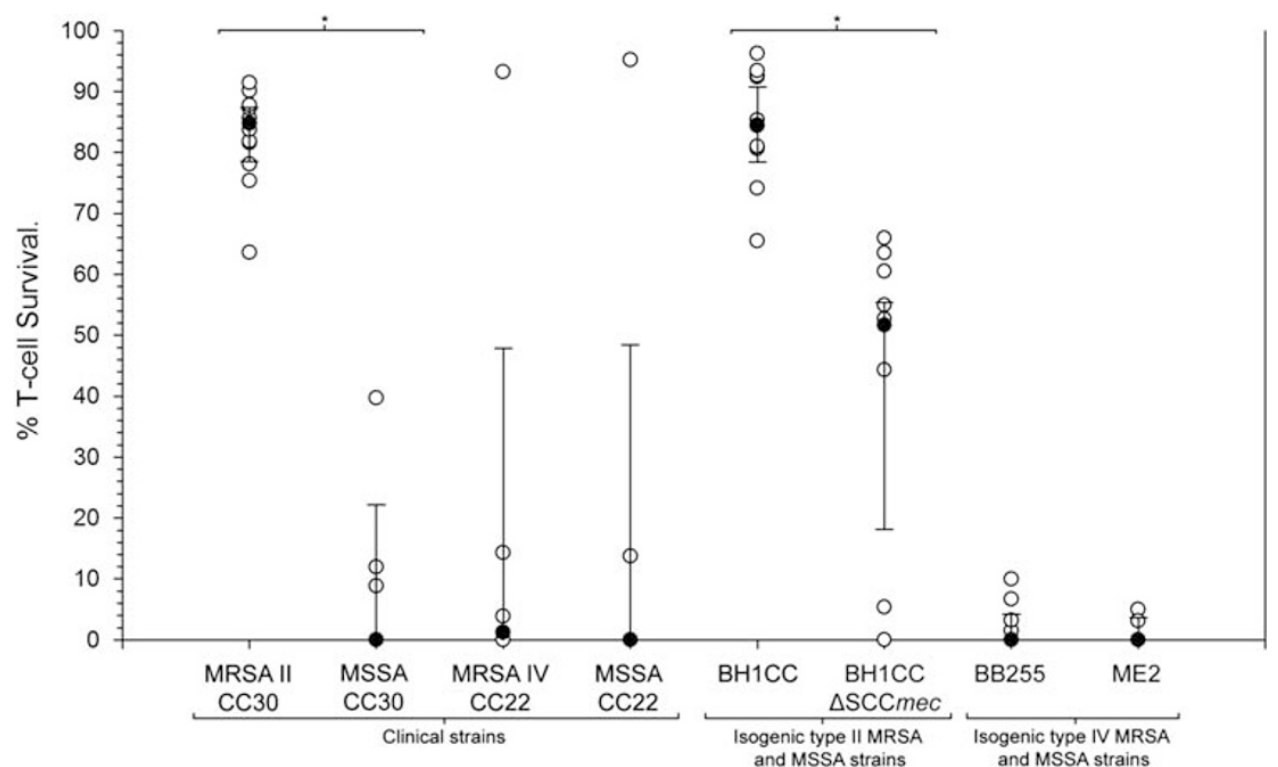

Figure 1 The type II but not the type IV SCCmec element affect $S$. aureus toxicity. Graph showing the percentage T-cell survival after exposure of T cells to $S$. aureus supernatant. The open circles represent data points, the filled circles show the median value and the error bars show the 95\% confidence intervals. The clinical MRSA (methicillin-resistant Staphylococcus aureus) strains were matched with clonally related MSSA (methicillin-sensitive Staphylococcus aureus) strains. The type II SCCmec carrying MRSAs (MRSAII) have significantly reduced toxicity compared with their related MSSAs (CC30s) $\left({ }^{*} P=0.0003\right)$. The type IV SCCmec carrying MRSAs (MRSAIV) were as toxic as their related MSSAs (CC22) $(P=0.5243)$, the Mann-Whitney test was used in both cases as data could not be normalized. The toxicity of the isogenic strains showed that BH1CC (MRSA) has median T-cell survival of $84 \%$ whereas its isogenic MSSA where the type II SCCmec element was excised (BH1CC $\Delta$ SCCmec)) was significantly more toxic with a median T-cell survival of $52 \%\left({ }^{*} P=0.0015\right)$. No difference was observed when the isogenic type IV SCCmec carrying MRSA (ME2) was compared with its MSSA ancestor (BB255) $(P=0.8600)$, the Mann-Whitney was test used in both cases as data could not be normalized. 
a

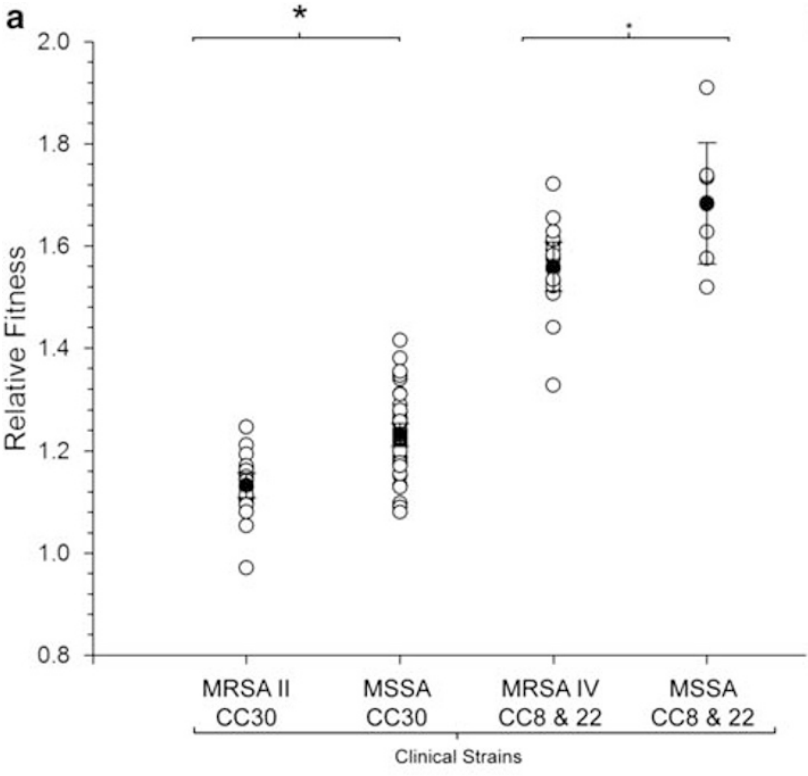

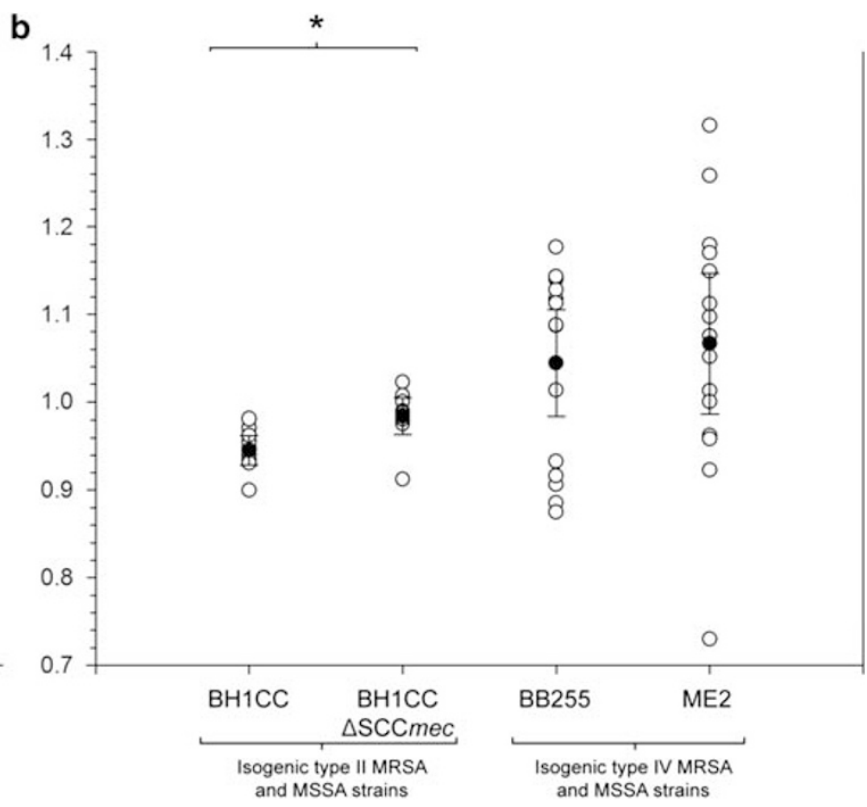

Figure 2 The type II but not the type IV SCCmec element affects the relative fitness of $S$. aureus. The open circles represent data points, the filled circles represent mean values and the error bars show the 95\% confidence intervals. (The * represent the statistically significant findings) (a) Graph showing the relative fitness of clinical S. aureus strains. The MRSAs (methicillin-resistant Staphylococcus aureus) have been matched with clonally related MSSAs (methicillin-sensitive Staphylococcus aureus). The relative fitness of both types of MRSAs is lower than their clonally related MSSAs $(P<0.0001, T=-6.08$, d.f. $=57$ and $P=0.046, T=2.36$, d.f. $=8$ for MRSAII and MRSAIV respectively). (b). Graph showing the relative fitness of isogenic MRSA and MSSA strains. The type II SCCmec MRSA (BH1CC) has reduced fitness relative to its isogenic MSSA strain BH1CC $\Delta$ SCCmec $(P=0.004, T=-3.28$, d.f. $=17)$. The fitness of the type IV MRSA strain, ME2, was not affected when compared to its isogenic MSSA strains BB255 $(P=0.64, T=-0.47$, d.f. $=26)$.

The Agr system in $S$. aureus is the major global regulator of toxin expression. To determine if toxin expression was also costly, we also measured the relative fitness of an isogenic pair of $\mathrm{Agr}^{+}$and $\mathrm{Agr}^{-}$ MSSA strains (RN6390B and RN6911 respectively; Ji et al., 1997) where the negative strain does not express toxins. These strains were competed against a marked MSSA strain, but the medium used was freshly spent broth in which the wild-type $\mathrm{Agr}^{+}$ strain was grown for $24 \mathrm{~h}$ to ensure maximal toxin expression from the residual Agr-encoded autoinducing peptide during the competition. The Agr mutant was significantly more fit than the toxin expressing wild-type strain (median relative fitness $\mathrm{Agr}^{+}, 1.36 ; \mathrm{Agr}^{-}, 1.48 ; P=0.003, T=-5.74$, d.f. $=9$, two-sample $t$-test), which shows that toxin expression is costly in environments in which there is no selection for it. Although other Agr-regulated factors may contribute to the fitness effects observed here, our previous work found it to be the most significant contributing factor to strain toxicity, and as such we consider it to be the major factor involved here.

Can the relative costs of toxin expression and antibiotic resistance explain the prevalence of different MRSAs in different environments?

Transmission of $S$. aureus between humans can be either symptomatic or asymptomatic (Massey et al., 2006). Asymptomatic transmission occurs by direct contact between a carrier and noncarrier.
Symptomatic transmission can also occur by direct contact but also by vector-mediated transmission through health-care workers. Symptomatic directcontact transmission, such as in cases of impetigo, results from the production of sticky pus-filled vesicles containing high densities of bacteria that get transmitted on fingers or during close-contact games such as rugby or American football ( $\mathrm{Lu}$ and Holtom, 2005). Vector-mediated transmission occurs in health-care settings between patients with underlying conditions that make them more susceptible to infection and where poor hand hygiene among health-care workers is prevalent. These diverse means of transmission have the potential to select for different bacterial characteristics resulting in diverse virulence phenotypes among $S$. aureus strains.

Mathematical modeling has been successfully used to help us understand transmission dynamics of many infectious diseases (Anderson and May, 1991). Here we use it to see if the relative costs associated with antibiotic resistance and toxin expression can explain why certain MRSA strains are restricted to health-care environments and others are not. We hypothesize that in the hospital environment the type II SCCmec-containing MRSA strains are successful because they are resistant to antibiotics and have offset some of the fitness costs associated with this by reducing costly toxin expression. They can survive with reduced toxin expression because transmission through 
health-care workers means they are not dependent on toxin-related production of transmissible material. They also have a population of compromised patients available with greater susceptibility to infection and so do not need to be as virulent to cause disease. This would explain why these MRSA strains have not been successful in the community setting where transmission through health-care worker and the prevalence of susceptible hosts are lower. We also hypothesize that the type IV SCCmec-containing MRSA strains are successful in both health-care and community settings because they have found an alternative means of reducing the fitness burden associated with being antibiotic resistant, which has allowed them to retain full toxicity.

To test our hypotheses and investigate whether these factors can explain the prevalence of these MRSA strains in different environments, we developed a mathematical model in which we competed three strain types (see Material and methods for full details). One had high-cost resistance and low-level (and therefore low-cost) toxin production, representative of type II SCCmec-containing MRSA strains. The second had low-cost resistance and high-level and high-cost toxin production representative of type IV SCCmec-containing MRSA strains. The third had no antibiotic resistance and high-level and highcost toxin expression representative of MSSA strains. Two environments were established, the first representative of the community setting where the majority of transmission is through direct rather than vector-mediated contact; the second representative of a hospital setting with a greatly enhanced chance of vector-mediated transmission but also a higher rate of treatment.

Figure 3 shows the time series for the proportion of the population infected with different $S$. aureus strains for the two different transmission settings: community (Figure 3a) and hospital (Figure 3b). The simulations started off with only MSSA being present in the population, and after it had reached a stable prevalence both MRSA types were introduced (at time $t=0$ ). Within the community (Figure 3a), the type IV MRSA quickly becomes the dominant strain because of increased toxicity and antibiotic resistance and despite a lower treatment success and increased lethality. In contrast, within a hospital setting (Figure 3b) the type II MRSA outcompetes the type IV MRSA because the now dominant secondary route of transmission (that is health-care workers) disadvantages enhanced toxicity and its associated fitness cost. However, the interaction between the two MRSAs here is dependent on the relative costs associated with the larger type II SCCmec element and the cost of toxin production, neither of which is empirically measurable. The true picture of $S$. aureus infection in hospitals is continually evolving and is currently slightly different from this model where MSSAs remain in a significant presence. We believe that this
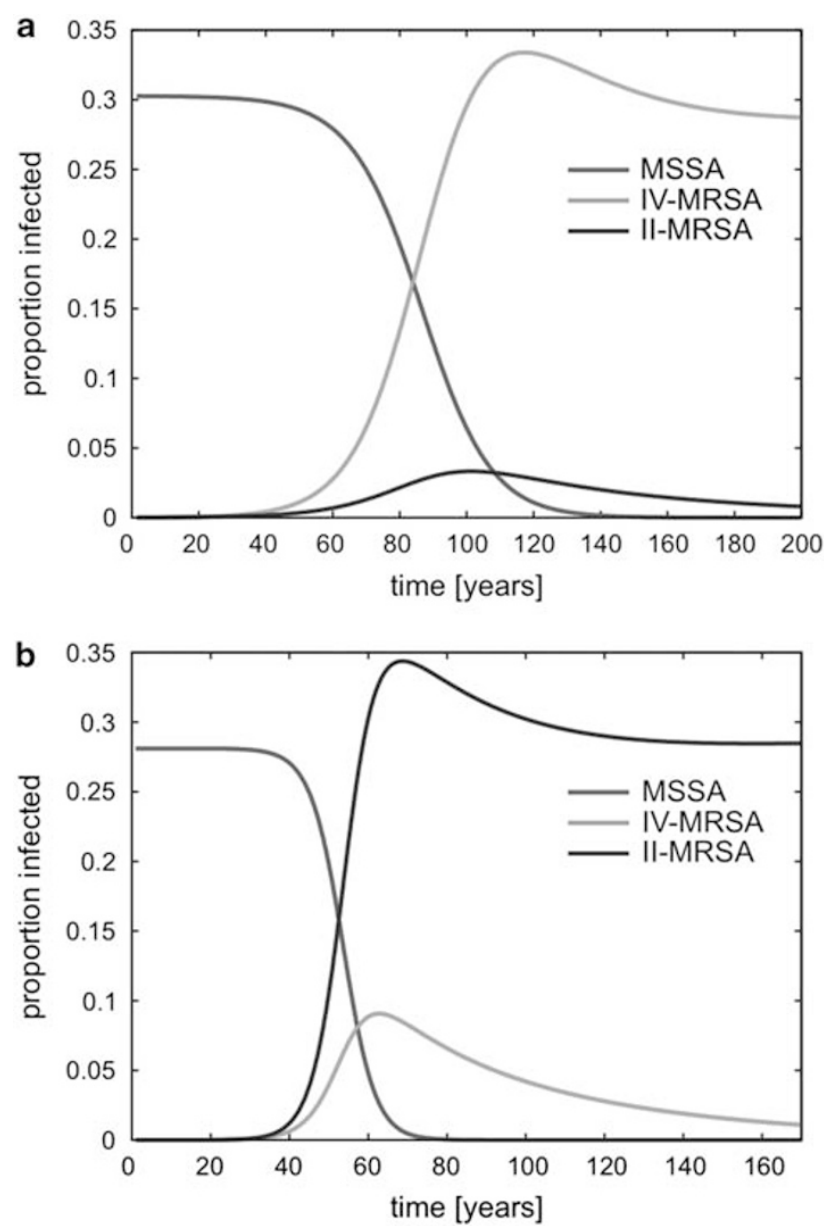

Figure 3 The relative costs of toxin expression and antibiotic resistance can explain the prevalence of MRSAs (methicillinresistant Staphylococcus aureus) in different environments. Graphical representation of a mathematical model where MSSAs (methicillin-sensitive Staphylococcus aureus), type IV SCCmec carrying MRSAs (IV-MRSA) and type II SCCmec carrying MRSAs (II-MRSA) are competed in two environments: (a) and (b). Environment (a) represents the community setting where the majority of transmission is through direct contact; and environment (b) represents a hospital setting with a greatly enhanced chance of vectored transmission but also a higher rate of treatment. In the community setting the type IV MRSAs become dominant, but in the hospital setting the type II MRSAs dominate.

reflects the infection of carriers with their own MSSA strain, and the continual reintroduction of MSSAs on carriers as they enter hospitals. Our experimental system was closed such that after the introduction of the MRSAs, no further bacteria were introduced into the system.

\section{Discussion}

Our findings and mathematical model reveal that the costs associated with toxin production and antibiotic resistance can explain why hospital- and community-acquired MRSA strains are successful in different environments where transmission routes 
and host susceptibility vary. We show that the large hospital-associated type II SCCmec element has a significant effect on the toxicity and growth rate of MRSAs. The mechanism by which the type II element reduces toxin expression is not fully understood but is possibly a consequence of the presence of the recently identified fudoh gene (Kaito et al., 2008). We believe the fitness burden associated with carrying the large type II SCCmec element is partially compensated for by the reduction in toxin expression, and that this contributes to the restriction of these MRSA strains to health-care settings where transmission is often through health-care workers and a large pool of susceptible hosts are available. The smaller type IV SCCmec element does not affect the relative fitness of the bacteria as much as the type II element, and does not affect toxicity resulting in a fully virulent, antibiotic-resistant bacterium. We believe that this may explain why these MRSA strains are successful both in healthcare settings and among healthy individuals in the community.

These findings suggest that in hospital environments transmission through health-care workers has significantly contributed to the evolution of this pathogen, allowing them to adapt to the fitness burdens associated with antibiotic resistance. Vector-mediated transmission is more traditionally associated with selecting for increased virulence, as the health of the host is not believed to contribute to their susceptibility to vectors (such as the transmission of malaria through mosquito). Here, however, the contrary seems to be true, where vector transmission appears to be selecting for decreased virulence. However in this instance the interaction of the host with its vector (health-care workers) is a secondary feature to infection. The patients are typically receiving the attention of health-care workers for treatment of underlying medical conditions or after surgery/trauma, so their immune system has already been compromised. The more this is compromised, the more attention they are likely to get from health-care workers and the more susceptible they are to infection. As such the dynamics are quite different to the traditional vector-transmitted diseases.

In our model of the hospital environment, the weighting given to the cost associated with unnecessary toxin production leads to the type II MRSAs eventually dominating. If however toxin production became an important part of the transmission of $S$. aureus in this environment, this dynamic would change. For example, if transmission through health-care workers was eliminated, the type IV MRSAs are likely to become dominant as their lowcost antibiotic resistance alongside high toxin production would give them a significant advantage.

The model of the community environment suggests that the type IV MRSAs become dominant and displace the MSSAs, however many other factors contribute to the success of this pathogen in the real world so it is difficult to say with any certainty what the future holds. What is interesting to consider is how asymptomatic transmission of $S$. aureus occurs among healthy people and how modern sanitized lifestyles puts up barriers to this. We previously hypothesized that it is barriers to asymptomatic transmission that have allowed $S$. aureus to become more virulent relative to $S$. epidermidis (Massey et al., 2006), and based on the work described here we suggest that it is possible that increased use of antibiotics and barriers to asymptomatic transmission of $S$. aureus between healthy people will continue to select for more virulent, antibioticresistant $S$. aureus strains.

\section{Acknowledgements}

This work was funded by studentships to JC (Stiefel) and JR (BBSRC). We thank Brigitte Berger-Bachi for providing strains and Angus Buckling and Sarah Turner for constructive discussion of the paper.

\section{References}

Anderson RM, May RM. (1991). Infectious Disease of Humans: Dynamics and Control. Oxford University Press: New York.

Andersson DI, Levin BR. (1999). The biological cost of antibiotic resistance. Curr Opin Microbiol 2: 489-493.

Collins J, Buckling A, Massey RC. (2008). Identification of factors contributing to T-cell toxicity of Staphylococcus aureus clinical isolates. J Clin Microbiol 46: 2112-2114.

Deurenberg RH, Vink C, Kalenic S, Friedrich AW, Bruggeman CA, Stobberingh EE et al. (2007). The molecular evolution of methicillin-resistant Staphylococcus aureus. Clin Microbiol Infect 13: 222-235.

Emmerson AM, Enstone JE, Griffin M, Kelsey MC, Smyth ET. (1996). The Second National Prevalence Survey of infection in hospitals-overview of the results. J Hosp Infect 32: 175-190.

Ender M, McCallum N, Adhikari R, Berger-Bachi B. (2004). Fitness cost of SCCmec and methicillin resistance levels in Staphylococcus aureus. Antimicrob Agents Chemother 48: 2295-2297.

Fowler Jr VG, Sakoulas G, McIntyre LM, Meka VG, Arbeit RD, Cabell CH et al. (2004). Persistent bacteremia due to methicillin-resistant Staphylococcus aureus infection is associated with agr dysfunction and low-level in vitro resistance to thrombininduced platelet microbicidal protein. J Infect Dis 190: 1140-1149.

Gordon RJ, Lowy FD. (2008). Pathogenesis of methicillinresistant Staphylococcus aureus infection. Clin Infect Dis 5: S350-S359.

Hiramatsu K, Cui L, Kuroda M, Ito T. (2001). The emergence and evolution of methicillin-resistant Staphylococcus aureus. Trends Microbiol 9: 486-493.

Ito T, Ma XX, Takeuchi F, Okuma K, Yuzawa H, Hiramatsu K. (2004). Novel type V staphylococcal cassette chromosome mec driven by a novel cassette chromosome recombinase, ccrC. Antimicrob Agents Chemother 48: 2637-2651. 
Ji G, Beavis R, Novick RP. (1997). Bacterial interference caused by autoinducing peptide variants. Science 276: 2027-2030.

Jones RN. (2003). Global epidemiology of antimicrobial resistance among community-acquired and nosocomial pathogens: a five-year summary from the SENTRY Antimicrobial Surveillance Program (19972001). Semin Respir Crit Care Med 24: 121-134.

Kaito C, Omae Y, Matsumoto Y, Nagata M, Yamaguchi H, Aoto $\mathrm{T}$ et al. (2008). A novel gene, fudoh, in the SCCmec region suppresses the colony spreading ability and virulence of Staphylococcus aureus. PLoS One 3: 3921.

Katayama Y, Ito T, Hiramatsu K. (2000). A new class of genetic element, staphylococcus cassette chromosome mec, encodes methicillin resistance in Staphylococcus aureus. Antimicrob Agents Chemother 44: 1549-1555.

Klevens RM, Morrison MA, Nadle J, Petit S, Gershman K, Ray S, et al., Active Bacterial Core surveillance (ABCs) MRSA Investigators. (2007). Invasive methicillin-resistant Staphylococcus aureus infections in the United States. JAMA 298: 1763-1771.

Labandeira-Rey M, Couzon F, Boisset S, Brown EL, Bes M, Benito Y et al. (2007). Staphylococcus aureus PantonValentine leukocidin causes necrotizing pneumonia. Science 315: 1130-1133.

Lee SM, Ender M, Adhikari R, Smith JM, Berger-Bächi B, Cook GM. (2007). Fitness cost of staphylococcal cassette chromosome mec in methicillin-resistant Staphylococcus aureus by way of continuous culture. Antimicrob Agents Chemother 51: 1497-1499.

Lenski R, Rose M, Simpson S, Tadler S. (1991). Long-term experimental evolution in Escherichia coli. I. Adaptation and divergence during 2,000 generations. Am Nat 138: 1315.

Li M, Diep BA, Villaruz AE, Braughton KR, Jiang X, DeLeo FR et al. (2009). Evolution of virulence in epidemic community-associated methicillin-resistant Staphylococcus aureus. Proc Natl Acad Sci USA 106: 5883-5888.

Lowy FD. (1998). Staphylococcus aureus infections. N Engl J Med 339: 520-532.
Lu D, Holtom P. (2005). Community-acquired methicillinresistant Staphylococcus aureus, a new player in sports medicine. Curr Sports Med Rep 4: 265-270.

Ma XX, Ito T, Tiensasitorn C, Jamklang M, Chongtrakool P, Boyle-Vavra S et al. (2002). Novel type of staphylococcal cassette chromosome mec identified in community-acquired methicillin-resistant Staphylococcus aureus strains. Antimicrob Agents Chemother 46: 1147-1152.

Maisnier-Patin S, Andersson DI. (2004). Adaptation to the deleterious effects of antimicrobial drug resistance mutations by compensatory evolution. Res Microbiol 155: $360-369$.

Massey RC, Horsburgh MJ, Lina G, Hook M, Recker M. (2006). The evolution and maintenance of virulence in Staphylococcus aureus: a role for host-to-host transmission? Nat Rev Microbiol 4: 953-958.

O’Neill E, Pozzi C, Houston P, Humphreys H, Robinson DA, Loughman A et al. (2008). A novel Staphylococcus aureus biofilm phenotype mediated by the fibronectin-binding proteins, FnBPA and FnBPB. J Bacteriol 190: 3835-3850.

Noto MJ, Fox PM, Archer GL. (2008). Spontaneous deletion of the methicillin resistance determinant, mecA, partially compensates for the fitness cost associated with high-level vancomycin resistance in Staphylococcus aureus. Antimicrob Agents Chemother 52: 1221-1229.

Salter RD, Cresswell P. (1986). Impaired assembly and transport of HLA-A and -B antigens in a mutant TxB cell hybrid. EMBO J 5: 943-949.

Vaudaux PE, Monzillo V, Francois P, Lew DP, Foster TJ, Berger-Bächi B. (1998). Introduction of the mec element (methicillin resistance) into Staphylococcus aureus alters in vitro functional activities of fibrinogen and fibronectin adhesins. Antimicrob Agents Chemother 42: 564-570.

Wang R, Braughton KR, Kretschmer D, Bach TH, Queck SY, Li M et al. (2007). Identification of novel cytolytic peptides as key virulence determinants for community-associated MRSA. Nat Med 13: 1510-1514 http:// saureus.mlst.net/.

Supplementary Information accompanies the paper on The ISME Journal website (http://www.nature.com/ismej) 